\title{
Computer Color Mapping of Configuration of Goss Grains after Transverse Cold Rolling in Grain Oriented Silicon Steel ${ }^{*}$
}

\author{
By Yukio INOKUTI,** Chizuko MAEDA $A^{* *}$ and Yo ITO**
}

\begin{abstract}
Synopsis
In order to elucidate the formation of Goss grains after transverse cold rolling in a grain oriented silicon steel, computer color mapping was performed by using orientation information of Kossel diffraction patterns taken in advance in the recrystallized grains.

For computer color mapping in the vicinity of the steel surface after first stage cold rolling in the transverse direction and the subsequent intermediate annealing, the formation of Goss grains is very scarce and the size of these primary grains is much larger than that after a first straight cold rolling. Also, for similar computer color mappings after a second stage cold rolling in the transverse direction and the subsequent decarburization and primary recrystallization annealing, the formation of Goss grains is strongly inhibited. This inhibition has a decisive influence in conjunction with the cold rolling directions in the first and second stages: the previously generated Goss grains disappear by the transverse cold rolling. The preferential formation and inheritance of the celebrated Goss nuclei, which can be inherited by the structure memory from the original hot rolled silicon steel, are considered to be accomplished in cold rolling parallel to the hot rolling direction.
\end{abstract}

Key words: grain oriented silicon steel; Kossel pattern; color mapping by computer; rolling direction; secondary recrystallization; orientation distribution.

\section{Introduction}

Potential nuclei for Goss secondary recrystallization are generated in the strain free, highly oriented Goss areas in the vicinity of the surface of hot rolled silicon steel. ${ }^{1-5)}$ These highly oriented Goss areas of secondary nuclei are inherited by the structure memory in the vicinity of the steel surface after the decarburization and primary recrystallization treatment, following the first cold rolling, intermediate annealing and the second cold rolling.

In the subsequent secondary recrystallization annealing, the colonies of primary recrystallized Goss grains in the vicinity of steel surface show preferential grow $^{1,6-8)}$ which is accomplished by a competitive growth of large primary Goss grains with the low angle boundaries. These colonies (secondary nuclei) consume the other primary recrystallized grains and grow into giant-size $(3 \sim 8 \mathrm{~mm})$ secondary grains.

It was important to ascertain whether this hypothesis for the nucleus formation mechanism; inheritance and preferential growth of the celebrated Goss secondary grains is well performed in actual conditions. For this purpose, an attempt was made to examine the formation of Goss grains after removing strong Goss textures by grinding both the surface of hot rolled sheet ${ }^{9)}$ and also after cold rolling in the trans- verse direction in the first and second stages. ${ }^{10)}$ It has been shown that the perfection of Goss secondary grains after treatment by these two methods is not satisfactory in comparison with the ordinary processing. Therefore, it is necessary to clarify the mechanism by which these two treatments influence the formation of Goss grains.

The object of the present work was to make computer color mapping of the configuration of Goss grains after transverse cold rolling in a grain oriented silicon steel. This was accomplished by use of the orientation information of Kossel diffraction patterns taken in advance for recrystallized grains.

\section{Specimen Preparation and Experimental Pro- cedure}

The starting materials used in the present investigation were hot rolled sheets ${ }^{10)}$ of about $2.7 \mathrm{~mm}$ thickness. The chemical composition of this material was C: $0.044 \%$, Si : $3.35 \%$, Mn: $0.070 \%$, Se: $0.020 \%$, Sb: $0.025 \%$ and Mo: $0.013 \%$. Recently, the silicon content in steel has been increased from 3.0 to $3.35 \%$, to minimize the iron loss in steel product. Molybdenum $^{11}$ has also been added to silicon steels to inhibit the intergranular fracturing during slab soaking or hot rolling, to strengthen the Goss texture in the vicinity of steel surface during hot rolling, and to obtain a thin and smooth film of forsterite $\left(\mathrm{Mg}_{2} \mathrm{SiO}_{4}\right)$ after final annealing. The significance of the addition of other inhibitors such as MnSe and $\mathrm{Sb}$ has already been described, ${ }^{12)}$ as well as the method for producing silicon steel containing these elements ${ }^{13}$ ) and the slab soaking and hot rolling methods. ${ }^{14)}$ The important points in the production of silicon steel are to keep impurities as low as possible, to decompose and dissolve all the selenides during slab soaking, and to provide a fine dispersion of $\mathrm{MnSe}$ precipitates during hot rolling.

To elucidate the effects of cold rolling direction on the formation of Goss texture, specimens were first cold rolled in the first stage about $70 \%$ reduction in the direction parallel ( $\mathrm{L}$ direction) or transverse $(\mathrm{C}$ direction) to the hot rolling direction. All specimens were intermediately annealed for $3 \mathrm{~min}$ at $950^{\circ} \mathrm{C}$ in an atmosphere of $50 \% \mathrm{H}_{2}+50 \% \mathrm{~N}_{2}$ mixed gas, and cold rolled in the second stage to the final thickness of $0.3 \mathrm{~mm}$. A diagram of these combinations of rolling direction in the first and second cold rollings of Specimens A D is shown in Fig. 1. After the

* Based on the paper presented to the 110th ISIJ Meeting, October 1985, S1352, at Niigata University in Niigata. Manuscript received on September 18, 1986; accepted in the final form on December 12, 1986. (C) 1987 ISIJ

** Iron \& Steel Research Laboratories, Kawasaki Steel Corporation, Kawasaki-cho, Chiba 260. 


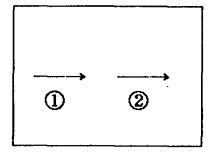

$\rightarrow$ H. R.

A

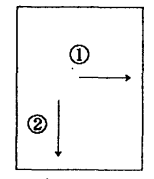

B

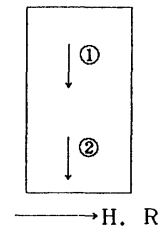

C

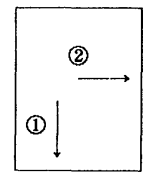

$\rightarrow$ H. R.

D
(1) The first cold rolling

(2) The second cold rolling

Fig. 1. Diagram of cold rolling direction in the first stage 1 and the second stage 2 .

decarburization and primary recrystallization annealing for $5 \mathrm{~min}$ at $820^{\circ} \mathrm{G}$ in wet $\mathrm{H}_{2}$ gas, the specimens were subjected to secondary recrystallization annealing for $50 \mathrm{~h}$ at $850^{\circ} \mathrm{C}$ in flowing $\mathrm{Ar}$ gas.

To elucidate the effects of the cold rolling direction on the formation of Goss texture, texture variations from the first cold rolling to decarburization and primary recrystallization annealing were measured at a depth of $1 / 10$ thickness of sheet below the steel surface and at the midsection by the transmission Kossel (TK) technique and pole figure method. The method of specimen preparation for pole figure method has already been reported ${ }^{5,6)}$; the preparation of a thin specimen for TK examination of the region just below the steel surface after an intermediate annealing or the decarburization and primary recrystallization annealing is given. Specimens of $0.8 \times 30$ $\times 30 \mathrm{~mm}^{3}$ after an intermediate annealing and those of $0.3 \times 30 \times 30 \mathrm{~mm}^{3}$ after the decarburization and primary recrystallization annealing were mechanically ground to about 180 and $70 \mu \mathrm{m}$ in thickness from one surface, respectively, chemically polished in a $3 \% \mathrm{HF}+$ $\mathrm{H}_{2} \mathrm{O}_{2}$ mixed solution until about $15 \mu \mathrm{m}$ thickness, and finally etched with $3 \%$ nital. Representative areas of the microstructure were selected and the orientations of primary grains were examined in detail by using a TK apparatus. ${ }^{15)}$ The details of such TK examination and analysis have been reported previously. ${ }^{16)}$

The computer color mapping of crystallographic orientation of the primary recrystallized grains after an intermediate annealing and the decarburization and primary recrystallization was performed by an image processing analyzer. Orientation analysis of Kossel diffraction patterns, which had been taken for each primary grain, was performed by using a computer chart. In the computer chart, angle $(\gamma)$, forming an indexed arbitrary diffraction plane relative to the rolling plane, and angle $(\psi)$ forming by the intersection of these two planes and the rolling direction, were drawn by the computer in advance. ${ }^{16-18)}$ The color mappings of primary recrystallized grains in terms of the orientations of normal and rolling directions (N.D. and R.D. $)^{17,18)}$ were prepared by an image analyzer (Luzex 5000 manufactured by Nireco Co.), ${ }^{19)}$ and directly photographed from the color display. Data $(\gamma, \phi)$, determined by a chart from Kossel diffraction patterns, was converted by computational processing into the parameters ${ }^{17)}$ necessary for an indication on a unit triangle of stereographic projection showing N.D. for the crystallographic plane and R.D. for the crystallographic direction. (The unit triangle is shown in each lower part of each color mapping.) Three primary colors, blue (001), red (011), and green (111) representing the crystallographic planes of grains were blended at linear 256 levels corresponding to the angle of deviation from each vertex. In a similar way, the three colors for [001], [011], and [111] for the crystallographic directions of grains were blended at logarithmic 256 levels. In the latter case, that is, for the crystallographic direction of grains the color blend at the logarithmic 256 levels was based on consideration of the preferential formation of primary grains of [001] alignment in the rolling direction. The boundary of all primary grains was classified by computational processing according to three categories in which the deviation angles of the N.D. and R.D. of mutual grains were photographed with the width of 1 pixel within $10^{\circ}$, 2 pixels within $25^{\circ}$, and 3 pixels for more than $25^{\circ}$ on the display. ${ }^{17)}$ This method of representation for the grain boundaries has been reported. ${ }^{17)}$ Further, in order to make the brightness of displayed colors uniform, the contrast enhance was averaged.

\section{Experimental Results}

\section{Formation of Texture due to the Difference of Cold Rolling Direction}

Figure 2 shows (100) pole figures at a depth of $1 / 10$ the sheet thickness under the steel surface after cold rolling of the first stage and subsequent intermediate annealing. Figure 3 shows textures measured similarly at the center of sheet thickness. The R.D. of the (100) pole figures in Figs. 2 and 3 denotes that of the first cold rolling, in which Specimens $G$ and D are different from the ordinal hot rolling direction. Figures 4 and 5 show (100) pole figures of a depth of $1 / 10$ the sheet thickness under the sheet surface and those of the center of the sheet thickness, respectively, after cold rolling of the second stage and subsequent decarburization and primary recrystallization annealing. Table 1 (p. 305) lists the principal texture components at a depth of $1 / 10$ thickness below the surface and at the center of thickness, under the various conditions seen in Specimens A D in Figs. 2 to 5 .

From Fig. 2 and Table 1, the principal texture components in the vicinity of surface after the first stage cold rolling in the ordinal direction and the subsequent intermediate annealing are $\{111\}<112\rangle$ and strong $\{h k 0\}\langle 001\rangle$, respectively, whereas those after the first stage cold rolling in the transverse direction and the subsequent intermediate annealing are $\{111\}\langle 110\rangle$, and $\{111\}\langle 112\rangle$ and $\{100\}\langle 011\rangle$, respectively. These texture variations are consistent with the experimental results obtained by the cold rolling and recrystallization of $\mathrm{Fe}-3 \% \mathrm{Si}$ single crystals. ${ }^{2,21)}$ As seen in Fig. 4 (p. 305) and Table 1, the principal texture components in the vicinity of surface after the second stage cold rolling of the ordinal direction (Specimen A) and the subsequent decarburization and recrystallization annealing are 


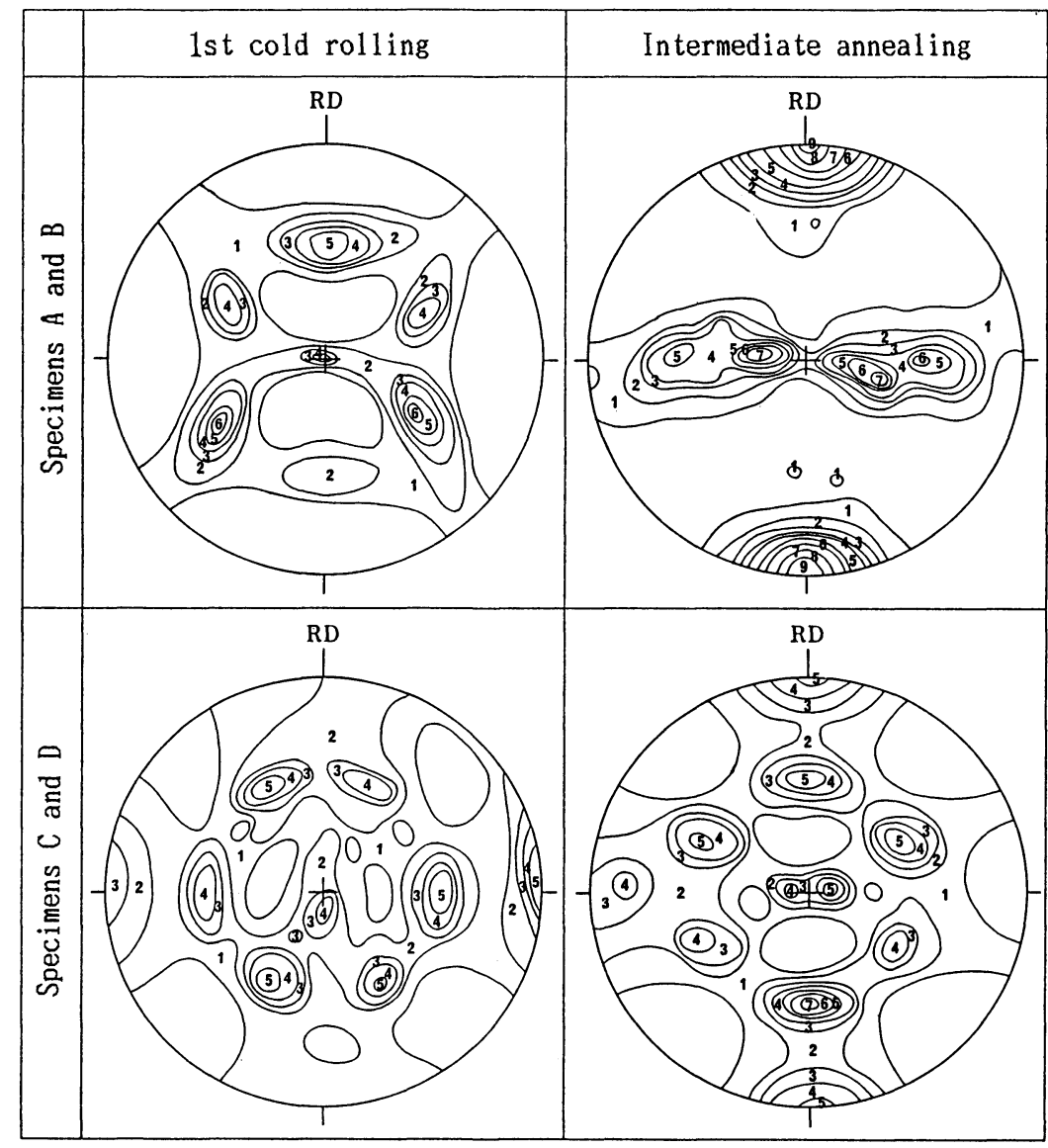

Fig. 2.

(100) pole figures at a depth of $1 / 10$ the sheet thickness under the steel surface of Specimens A $\mathrm{D}$ after cold rolling of the first stage and intermediate annealing.
Fig. 3.

(100) pole figures at the center of the steel sheet of Specimens $A \sim D$ after cold rolling of the first stage and intermediate annealing.

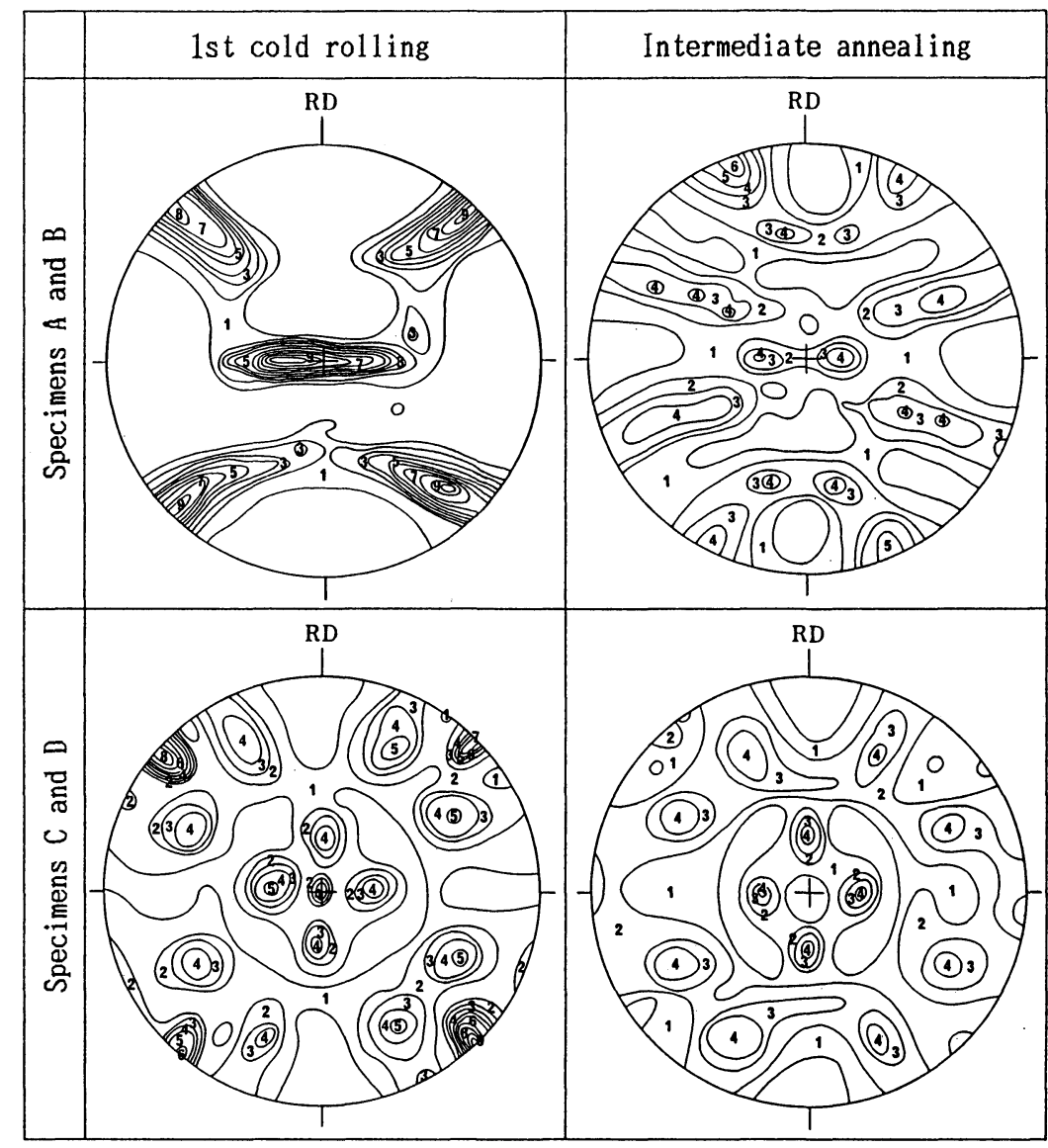


Table 1. Principal texture components at a depth of $1 / 10$ the sheet thickness under the steel surface and at the center of the steel sheet in Specimens A D.

\begin{tabular}{|c|c|c|c|c|}
\hline & 1st cold rolling & Intermediate annealing & 2nd cold rolling & $\begin{array}{c}\text { Decarburization and } \\
\text { primary recrystallization } \\
\text { annealing }\end{array}$ \\
\hline $\begin{array}{l}\text { Specimen A } \\
\text { (Standard) }\end{array}$ & $S\{111\}\langle 112\rangle$ & $S$ Strong $\{h k 0\}\langle 001\rangle$ & $\begin{array}{l}S \quad\{111\}\langle 112\rangle \\
\quad\{100\}\langle 011\rangle \\
C\langle 011\rangle \text { fiber texture }\end{array}$ & $\begin{array}{l}S \text { Strong }\{h k 0\}\langle 001\rangle \\
C\{110\}<001\rangle \\
\quad\{11\}<112\rangle\end{array}$ \\
\hline $\begin{array}{l}\text { Specimen B } \\
(\mathrm{L} \rightarrow \mathrm{C})^{*}\end{array}$ & $\begin{array}{l}C \text { Strong } \\
\langle 011\rangle \text { fiber texture }\end{array}$ & $\begin{aligned} & C\{001\} \sim\{126\} \\
&\langle 210\rangle\end{aligned}$ & $\begin{array}{l}S\{100\}\langle 011\rangle \\
\quad\{110\}\langle 112\rangle \\
C\langle 011\rangle \text { fiber texture }\end{array}$ & $\begin{aligned} S & \{111\}<011\rangle \\
& \{133\}<611\rangle \\
C & \{110\}<001\rangle \\
& \{111\}\langle 112\rangle\end{aligned}$ \\
\hline $\begin{array}{l}\text { Specimen } \mathrm{G} \\
(\mathrm{C} \rightarrow \mathrm{C})^{*}\end{array}$ & $S\{111\}\langle 110\rangle$ & $\begin{aligned} S & \{111\}<112\rangle \\
& \{100\}<011\rangle\end{aligned}$ & $\begin{array}{l}S\{111\}<112\rangle \\
C\langle 011\rangle \text { fiber texture }\end{array}$ & $\begin{aligned} S & \{110\}<001\rangle \\
& \{111\}\langle 012\rangle \sim\langle 110\rangle \\
C & \{h k 0\}\langle 001\rangle\end{aligned}$ \\
\hline $\begin{array}{l}\text { Specimen } \mathrm{D} \\
(\mathrm{C} \rightarrow \mathrm{L})^{*}\end{array}$ & $\begin{array}{r}C \quad\{100\}<011\rangle \\
\quad\{421\}\langle 012\rangle\end{array}$ & $C\{421\}\langle 012\rangle$ & $\begin{aligned} S & \{111\}\langle 110\rangle \\
& \{100\}\langle 011\rangle \\
C & \langle 011\rangle \text { fiber texture }\end{aligned}$ & $\begin{array}{c}S \text { Strong }\{111\}<112\rangle \\
C \text { Weak }\{111\}<112\rangle \\
\text { Weak }\{410\}\langle 140\rangle\end{array}$ \\
\hline
\end{tabular}

$S$ : Surface, $C$ : Center

* See diagram of cold rolling direction in the first stage 1 and the second stage 2 in Fig. 1.

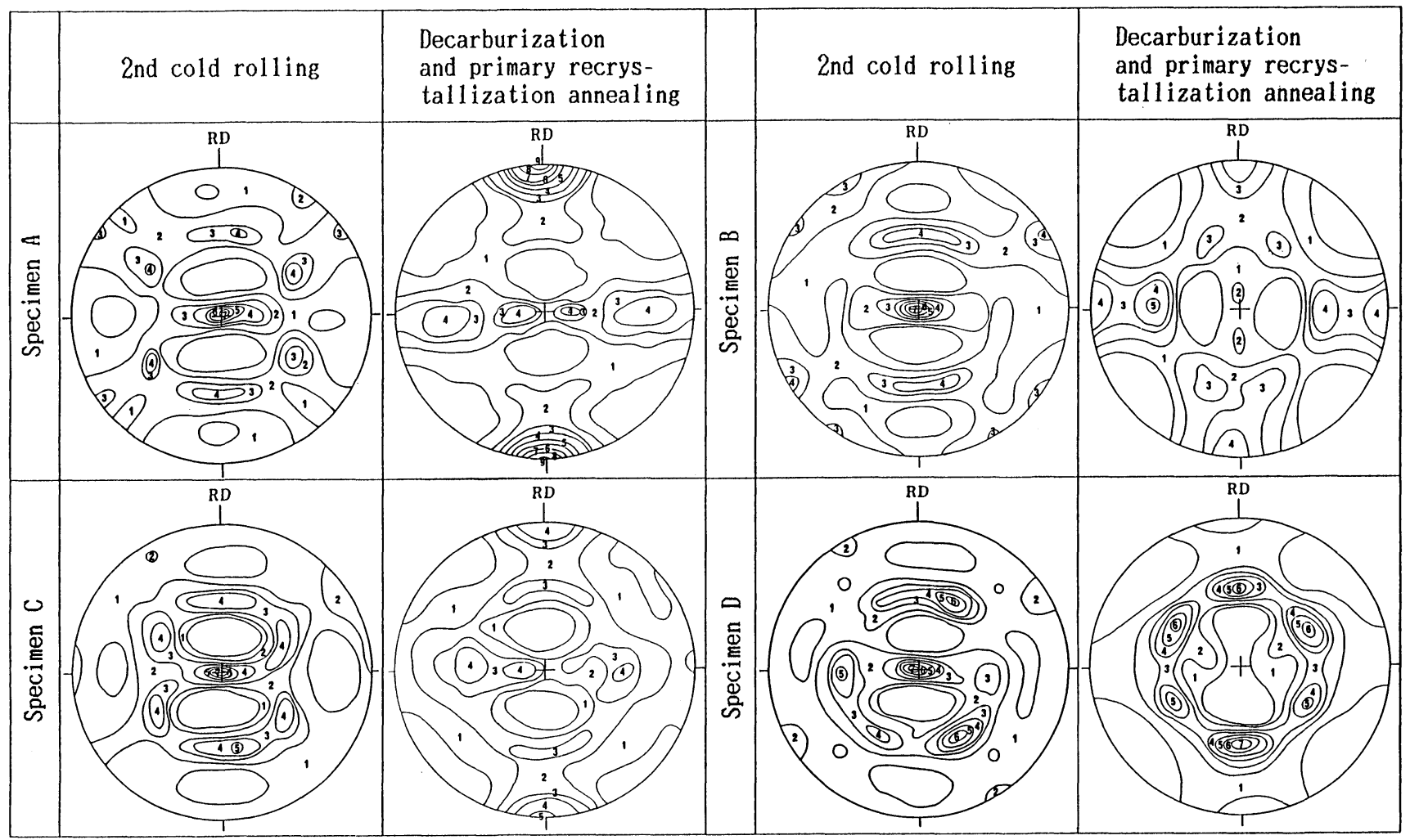

Fig. 4. (100) pole figures of a depth of $1 / 10$ the sheet thickness under the steel surface of Specimens A D after cold rolling of the second stage, and decarburization and primary recrystallization annealing.

$\{111\}<112\rangle$ and strong $\{h k 0\}\langle 001\rangle$, respectively. This texture variation is in good agreement with the experimental results, ${ }^{9,10,18)}$ that have been done to clarify the inheritance mechanism by the structure memory of Goss texture from the original hot rolled silicon steels. The principal texture components in the vicinity of surface after the second stage cold rolling are $\{100\}\langle 011\rangle$ and $\{110\}\langle 112\rangle$ for Specimen
$\mathrm{B},\{111\}\langle 112\rangle$ for Specimen $\mathrm{G}$, and $\{111\}\langle 110\rangle$ and $\{100\}\langle 011\rangle$ for Specimen D, whereas those after decarburization and recrystallization annealing are $\{111\}\langle 011\rangle$ and $\{133\}\langle 611\rangle$ for Specimen B, $\{110\}$. $\langle 001\rangle$ and $\{111\}\langle 112\rangle \sim\langle 110\rangle$ for Specimen $\mathrm{C}$, and strong $\{111\}\langle 112\rangle$ for Specimen D. It should be noted that the texture in the vicinity of the surface of Specimens A and $\mathrm{C}$ after decarburization and 
primary recrystallization annealing, develops to permit the development of Goss secondary grains, in a similar way, but that the Goss component which consists $\{h k 0\}\langle 001\rangle$ component $\mathrm{A}$ is much stronger in Specimen A than in Specimen G. As a result, perfection of the Goss secondary grains in Specimen A is more advanced than that in Specimen C. ${ }^{10}$

On the other hand, as can be seen in Fig. 3 and Table 1, the principal texture components at the center of thickness after the first stage cold rolling in the ordinal direction and the subsequent intermediate annealing are a strong $\mathrm{RD} / /\langle 011\rangle$ fiber texture, and $\{001\} \sim\{126\}\langle 210\rangle$, respectively, whereas those after the first stage cold rolling in the transverse direction and the subsequent intermediate annealing are $\{100\}$. $\langle 011\rangle$ and $\{421\}\langle 012\rangle$, and $\{421\}\langle 012\rangle$, respectively. In the second cold rolling and subsequent decarburization and primary recrystallization annealing shown in Fig. 5, the texture formed in the center of the sheet thickness manifests no noticeable variations which could be attributed to the difference of cold rolling direction; such variation, on the other hand, can be seen in the vicinity of surface.

\section{Computer Color Mapping}

1. Computer Color Mapping of Primary Recrystallized Grains after the First Stage Cold Rolling in the Transverse Direction and Subsequent Intermediate Annealing

Figures 6 and 7 show computer color mappings of the N.D. and R.D., respectively, of the primary recrystallized grains after cold rolling of first stage in the transverse direction and subsequent intermediate annealing. From Figs. 6 and 7 there is no formation of Goss grains, where large primary grains of $\{110\}$. $\langle 112\rangle,\{100\}\langle 001\rangle$ and $\{100\}\langle 011\rangle$ orientations are formed. Also, the size of primary grains in this area is several times as that in straight cold rolling. The grain boundary of each grain manifests a large grain boundary width of 3 pixels for more than $25^{\circ}$. It should be noted that cold rolling in the transverse direction and subsequent intermediate annealing inhibits the formation of Goss grains and weakens the inhibition of the normal grain growth during an intermediate annealing.

\section{Computer Color Mapping of Primary Recrystallized Grains after Decarburization and Primary Recrystal- lization Annealing}

Figures 8 and 9 (p. 308) show computer color mappings of the N.D. and R.D., respectively, of the primary recrystallized grains at a depth of $1 / 10$ the sheet thickness under the steel surface after the treatment of Specimen $\mathrm{G}$ in Fig. 1. It is evident from Figs. 8 and 9 that the colonies of Goss grains form in a diagonally elongated area with about $200 \mu \mathrm{m}$ in width, and the grain boundaries of each Goss grain in this elongated area possess not only low angle boundaries, but also high angle boundaries. The formation of Goss grains in Specimen $\mathrm{G}$ is quite different from that in Specimen $\mathrm{A}^{18)}$, where Goss grains form preferentially in defined and elongated areas toward the rolling direction with the low angle boundaries within $10^{\circ}$.

Figures 10 and 11 show the respective computer

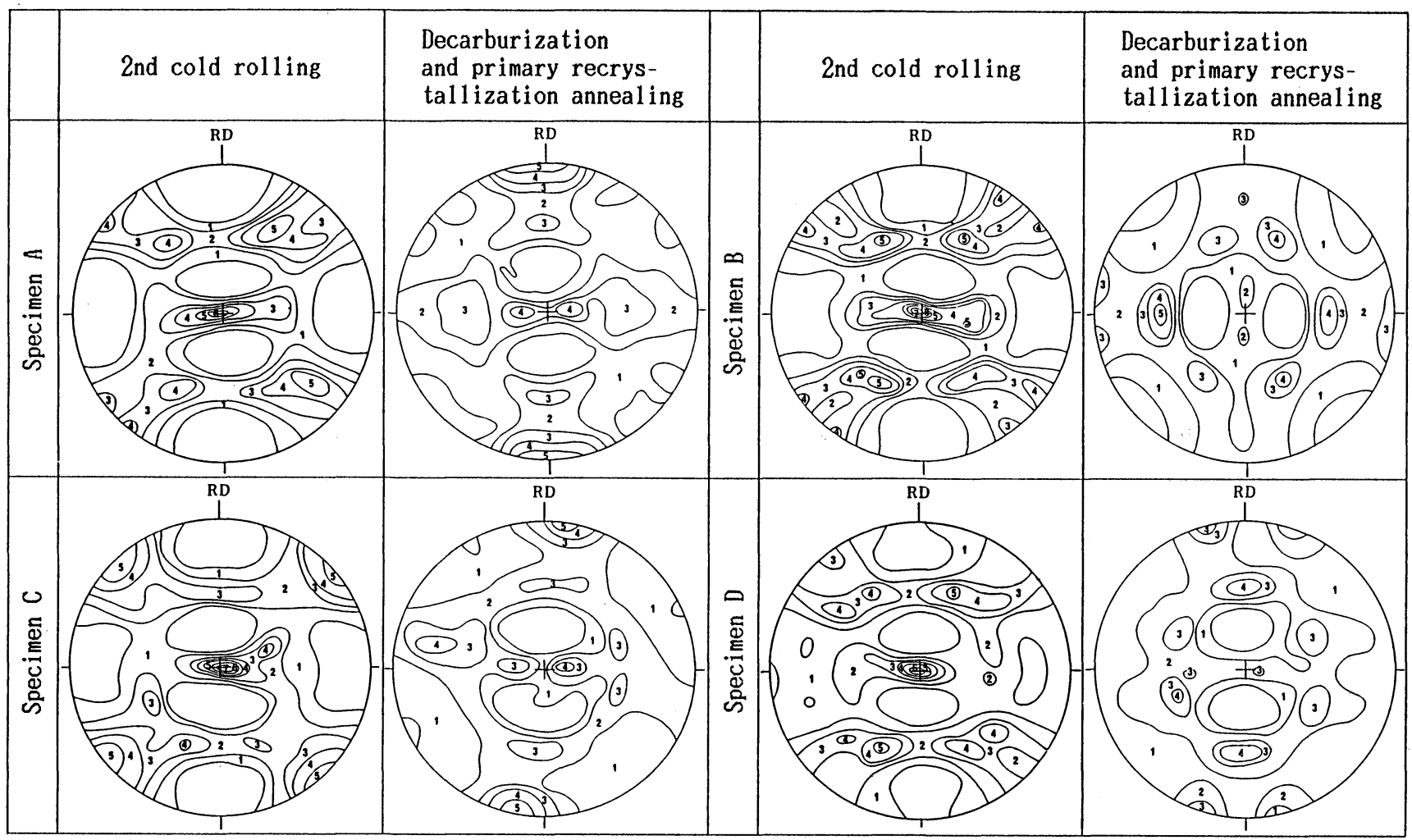

Fig. 5. (100) pole figures at the center of the steel sheet of Specimens $A \sim D$ after cold rolling of the second stage, and decarburization and primary recrystallization annealing. 


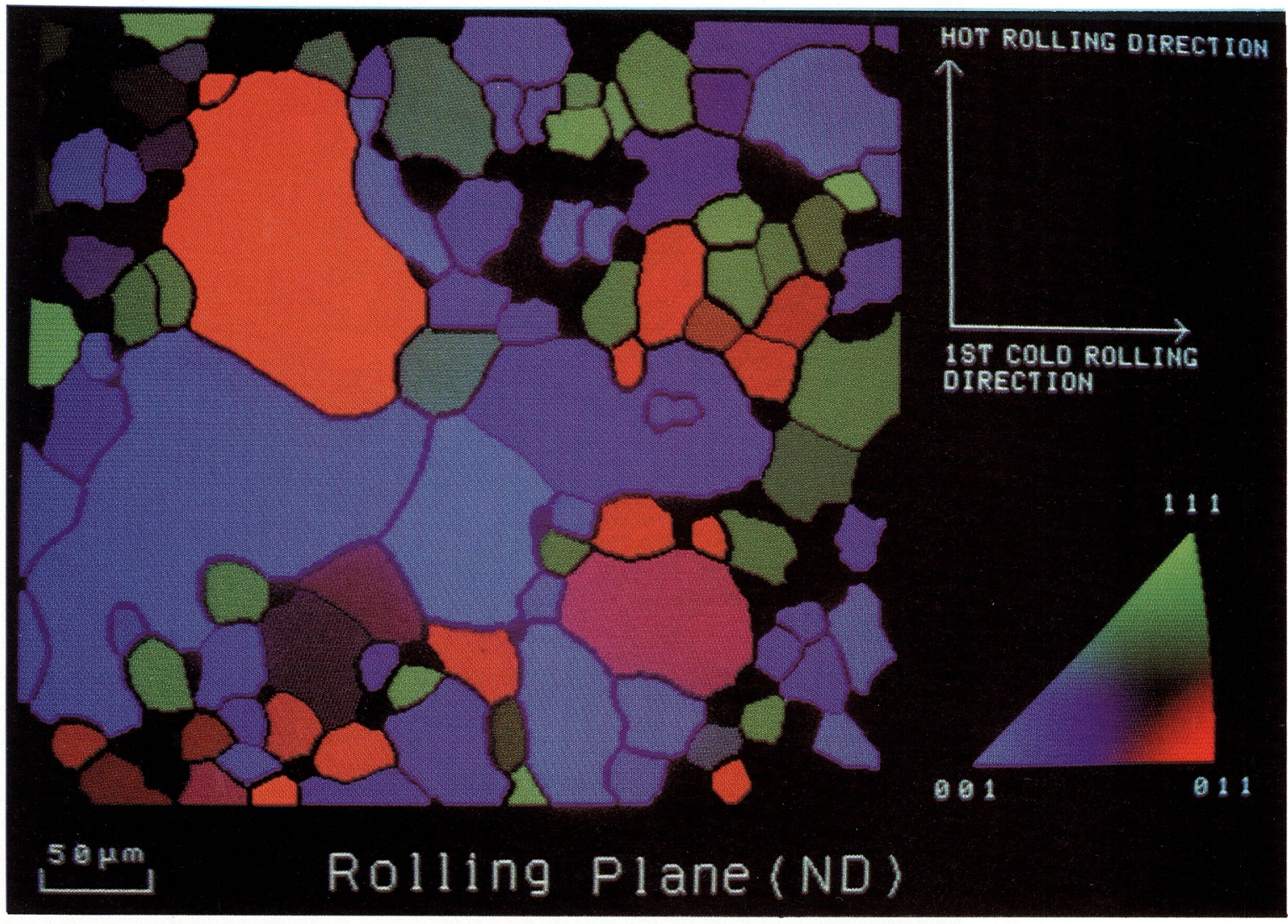

Fig. 6. Computer color mapping of N.D. of the primary recrystallized grains at a depth of $1 / 10$ the sheet thickness under the steel surface after cold rolling of the first stage in the transverse direction and subsequent intermediate annealing.

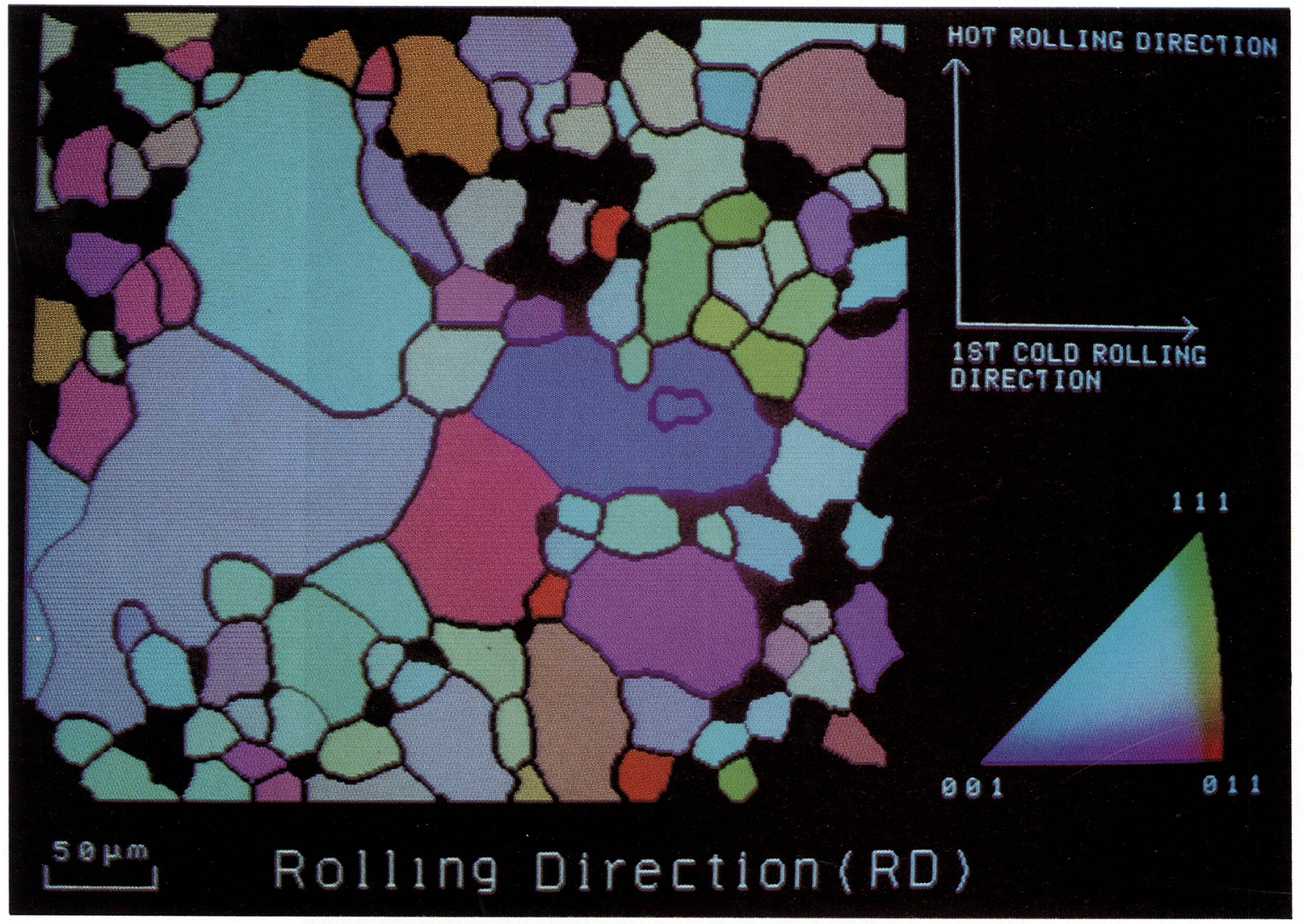

Fig. 7. Computer color mapping of R.D. of the primary recrystallized grains at a depth of $1 / 10$ the sheet thickness under the steel surface after cold rolling of the first stage in the transverse direction and subsequent intermediate annealing. 
Fig. 8.

Computer color mapping of N.D. of primary recrystallized grains at a depth of $1 / 10$ the sheet thickness under the steel surface after the treatment of Specimen C in Fig. 1.

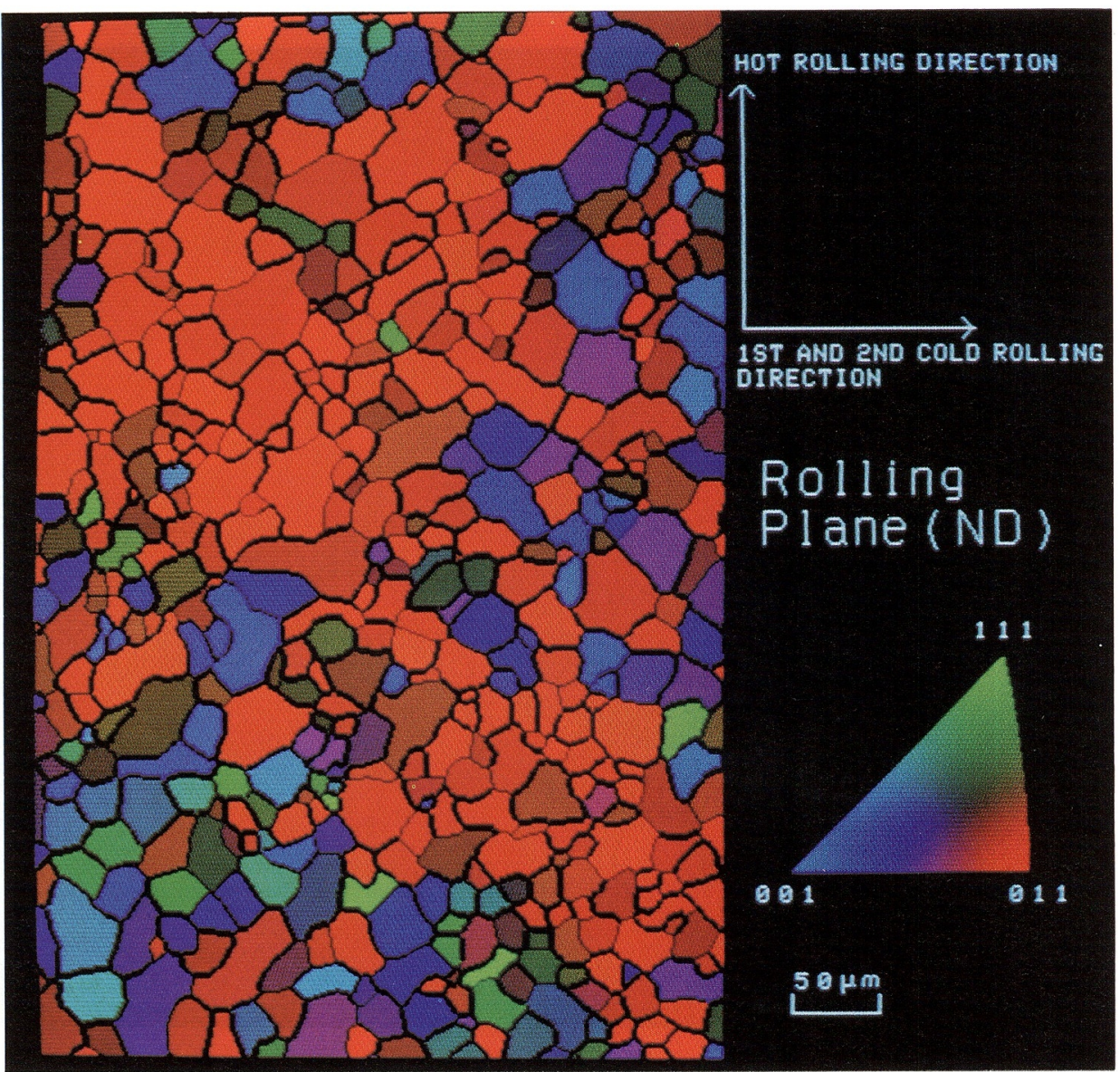

HOT ROLLING DIRECTION

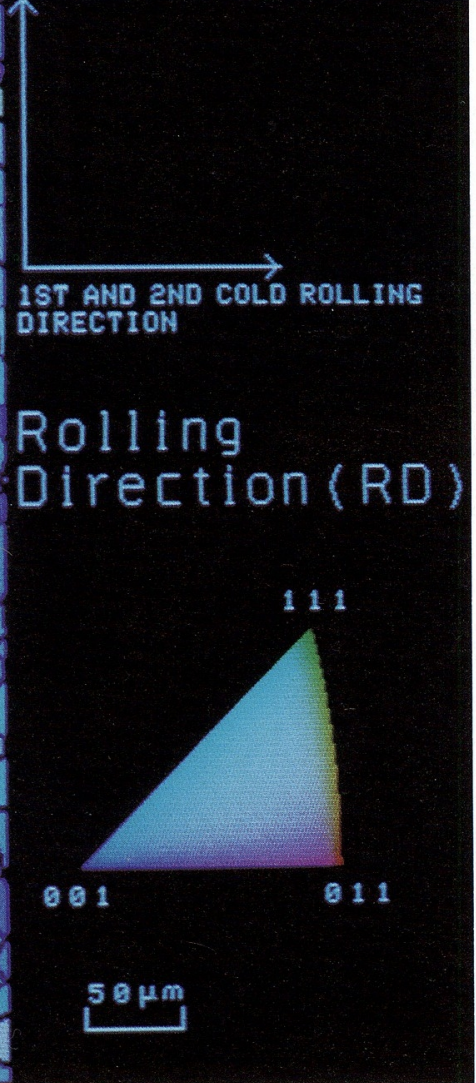

Fig. 9.

Computer color mapping of R.D. of primary recrystallized grains at a depth of $1 / 10$ the sheet thickness under the steel surface after the treatment of Specimen $\mathrm{C}$ in Fig. 1. 


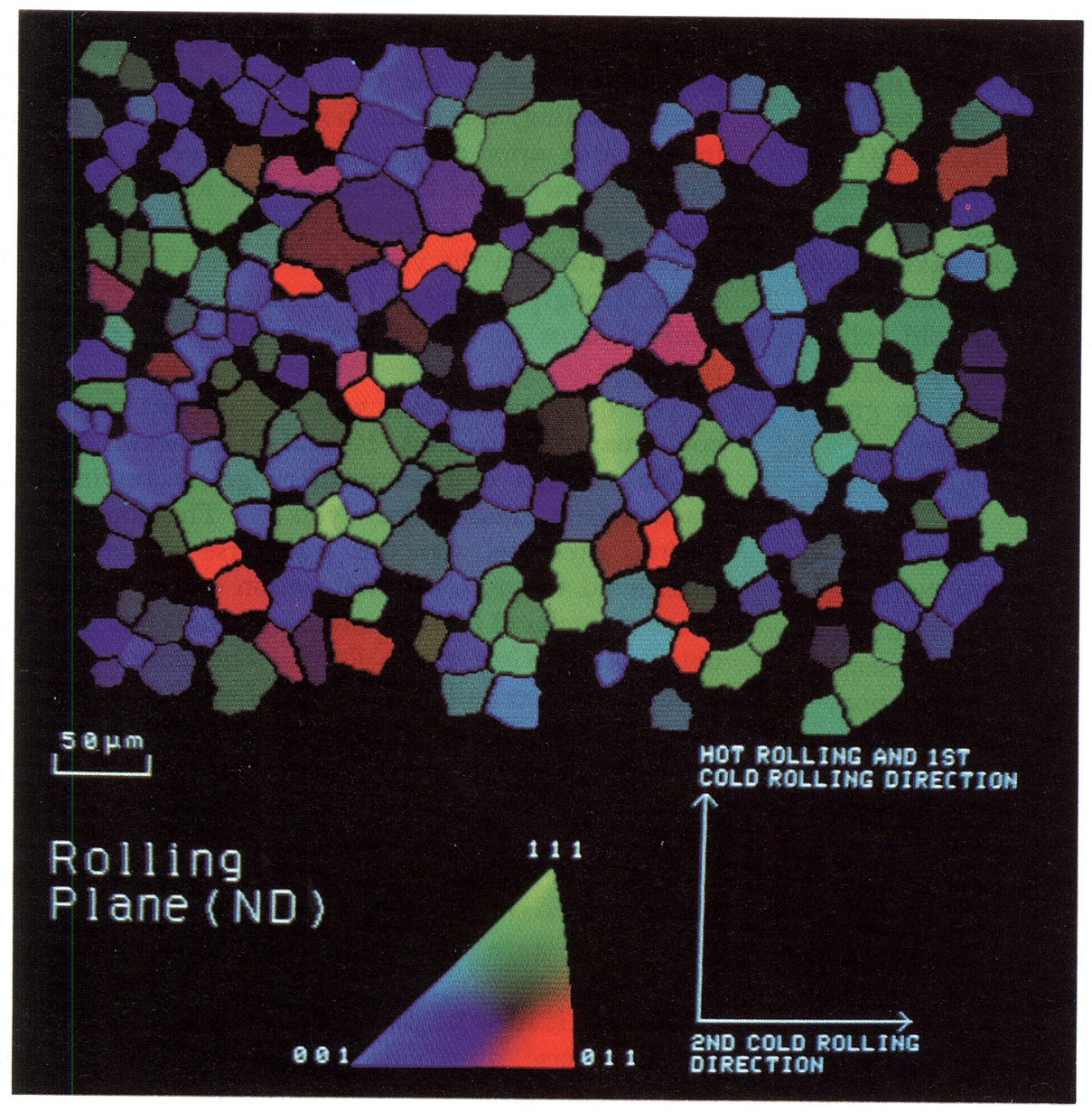

Fig. 10.

Computer color mapping of R.D. of primary recrystallized grains at a depth of $1 / 10$ the sheet thickness under the steel surface after the treatment of Specimen B in Fig. 1.
Fig. 11.

Computer color mapping of R.D. of primary recrystallized grains at a depth of $1 / 10$ the sheet thickness under the steel surface after the treatment of Specimen B in Fig. 1.

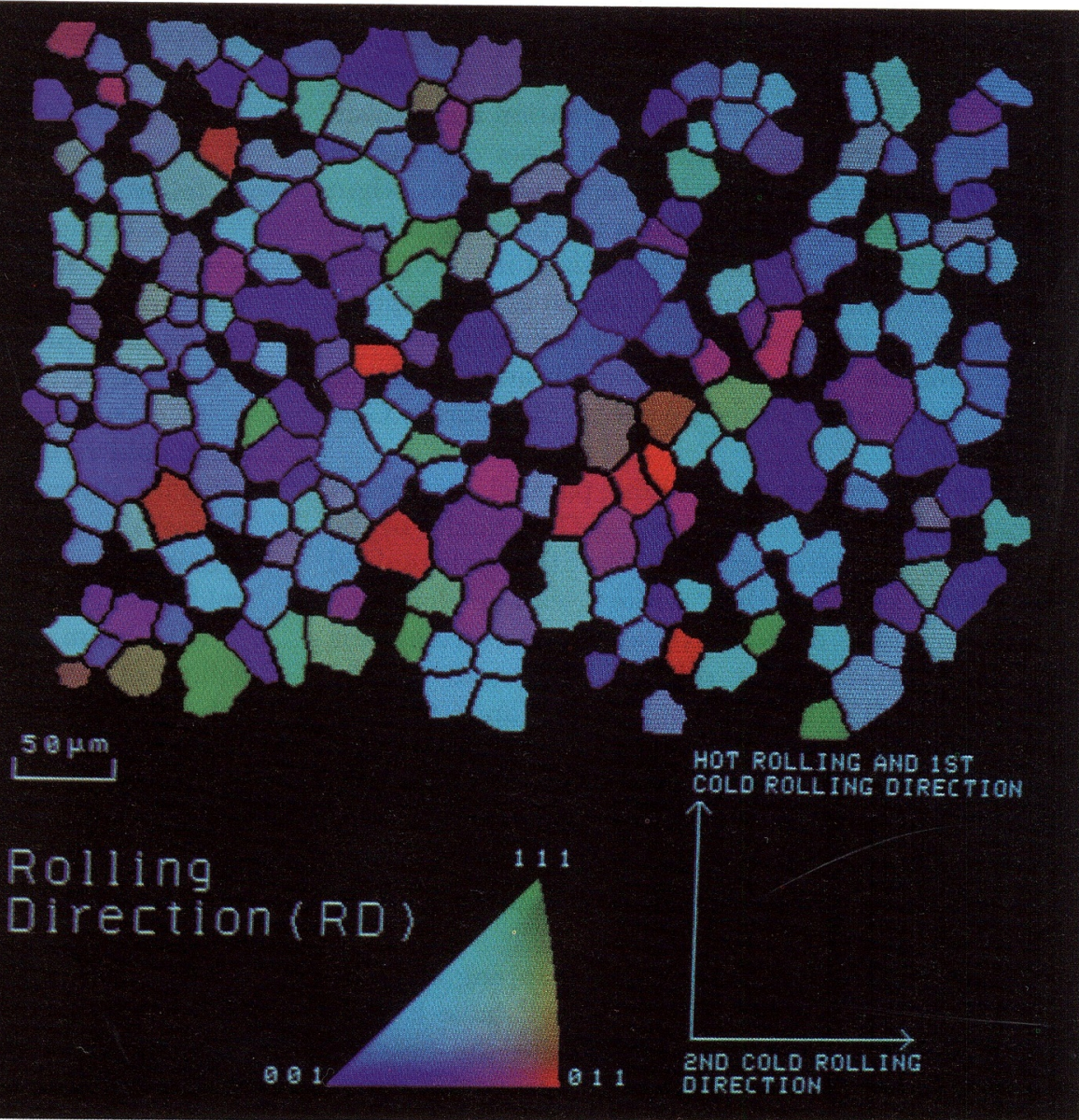


color mappings of the N.D. and the R.D. axies of the primary recrystallized grains at a depth of $1 / 10$ thickness below the surface after the treatment in Specimen B in Fig. 1. As will be seen in Figs. 10 and 11, formation of Goss grains is scarce and many grains with $\{111\}\langle 112\rangle$ orientation are formed. The Goss grains in this area occurs isolated, making high angle boundaries as wide as 3 pixels for more than $25^{\circ}$. It should be noted that alternate cold rolling in the transverse direction in Specimen B makes it to decrease drastically the formation of Goss grains in the vicinity of surface of silicon steel sheet.

\section{Discussion}

It has been reported that the perfection of Goss secondary grains is inhibited by removing both sides having two strong Goss textures in the vicinity of surface of hot rolled sheet ${ }^{9}$ and by the first or second stage cold rolling in the transverse direction. ${ }^{10}$ Because there is no available experimental technique to measure the orientation of small areas of 5 to $10 \mu \mathrm{m}$ and the small strains in primary and secondary grains, no experimental evidence to allow confident assertions concerning the formation of Goss grains has been obtained.

In the previous TK experiments of each process from the hot rolling to finished product of secondary grains, ${ }^{1-9)}$ the origin of Goss secondary grains has been found to be small strain-free areas with accurate Goss orientation where the Goss nuclei, 100 to $300 \mu \mathrm{m}$ wide and 100 to $1000 \mu \mathrm{m}$ long, are surrounded by small and accurate Goss areas (polygonized grains) elongated in the rolling direction at a depth of about $1 / 10$ thickness below the surface of the hot rolled sheet. ${ }^{2-4,7)}$ With respect to the formation mechanism of nuclei of secondary grains with Goss orientation which is unstable in the cold rolled state, the origin of nuclei of Goss secondary grains has been considered to be well protected by the polygonized matrix grains of Goss orientation. If an egg is taken as a metaphor, the "white" of an egg is the strained and polygonized matrix grains of Goss orientation and the "yolk" is the small strain-free areas of accurate Goss orientation.

In the subsequent cold rolling parallel to the hot rolling direction the small areas of Goss nuclei of the "yolk", which are protected by the polygonized Goss matrix grains of the "white", can be elongated in the direction of cold rolling by preserving the original state of as hot rolled. This state can be inherited until the decarburization and primary recrystallization annealing prior to the secondary recrystallization treatment, throughout the first cold rolling, intermediate annealing and second cold rolling. This inheritance mechanism of nuclei of Goss secondary grains has been designated as the "structure memory". ${ }^{1-8)}$

In the present computer color mapping in Figs. 8 and 9, the Goss areas of the "yolk" have formed in a diagonally elongated area due to the absence of a protective polygonized Goss matrix; this structure, the "white" of the metaphorical egg has been broken in the first stage cold rolling in the transverse direction. Because the Goss orientation is unstable in cold rolling, the grain boundaries of Goss grains in this area after an intermediate annealing possess not only low angle boundaries but also high angle boundaries. Therefore, the preferential formation of Goss grains in the vicinity of surface is considered to be strongly inhibited by destruction of the polygonized Goss matrix grains in the transverse cold rolling.

The question remains why the present computer color mappings in Figs. 10 and 11 indicate absence of the colonies of Goss primary grains retaining a trace of Goss areas of the "yolk". The double cold rolling alternately in the transverse direction are considered to cause the destruction of the formation zones of nuclei of Goss secondary grains; consequently only isolated Goss grains form. Therefore, in order to inherit successfully to the nuclei of Goss secondary grains from the original hot rolling up to the decarburization and primary recrystallization treatment, it is indispensable to cold roll in the same direction as the hot rolling. In addition, in such cases, the steel surface prior to cold rolling should be preserved in the original state without a treatment which weakens the Goss texture such as the grinding of steel surface. ${ }^{91}$

Finally, it is to be pointed out that cold rolling in the transverse direction weakens the inhibition of normal grain growth during an intermediate annealing. As the normal grain growth is influenced by the inhibitors in silicon steel, detailed study to clarify this problem is needed.

\section{Conclusions}

The results of the present study can be summarized as follows.

(1) Computer color mapping of primary recrystallized grains after cold rolling in the transverse direction and subsequent annealing in a grain oriented silicon steel provides the detailed information on the formation of Goss grains.

(2) In computer color mapping after the first stage cold rolling in the transverse direction and subsequent intermediate annealing, the formation of Goss grains is very scarce and the size of these primary grains is much larger than that for the first straight cold rolling.

(3) From computer color mapping after cold rolling of second stage in the transverse direction and subsequent decarburization and primary recrystalization annealing, the formation of Goss grains is found to be strongly inhibited. This inhibition has a decisive influence in conjuction with the cold rolling direction at the first and second stages; the generation of Goss grains is destructed by the transverse cold rolling.

(4) The preferential formation and inheritance of celebrated Goss nuclei, which can be inherited by the structure memory from original hot rolled silicon steel, can be accomplished in the cold rolling parallel to the hot rolling direction. 


\section{REFERENCES}

1) Y. Inokuti, Y. Simizu, C. Maeda and H. Shimanaka: Proc. 1st Risø Int'l Symp. on Metallurgy and Materials Science, Risø National Laboratory, Denmark, (1980), 70.

2) Y. Inokuti, C. Maeda, Y. Ito and H. Shimanaka: Trans. ISIJ, 23 (1983), 440.

3) Y. Inokuti and G. Maeda: Trans. ISIJ, 24 (1984), 655.

4) Y. Inokuti, C. Maeda and Y. Ito: Trans. ISIJ, 25 (1985), 233.

5) Y. Inokuti, C. Maeda and Y. Ito: Met. Trans., 16A (1985), 1613.

6) Y. Inokuti, C. Maeda, Y. Ito and H. Shimanaka: Proc. of The Sixth Int'l Conf. on Textures of Materials, II, ISIJ, Tokyo, (1982), 948.

7) Y. Inokuti, G. Maeda and Y. Ito: Tetsu-to-Hagané, 70 (1984), 2033.

8) Y. Inokuti: Tetsu-to-Hagané, 70 (1984), 2033.

9) Y. Inokuti, G. Maeda and T. Tanaka: J. Japan Inst. Metals, 49 (1985), 417.

10) Y. Inokuti, C. Maeda and Y. Ito: Proc. 4th Japan Inst. of Metals Int'l Symp. (JIMIS-4), Japan Inst. Metals, Sendai, (1985), 555.
11) Y. Inokuti and Y. Ito: Bull. Japan Inst. Metals, 23 (1984), 276.

12) I. Goto, I. Matoba, T. Imanaka, T. Gotoh and T. Kan: Proc. Soft Magnetic Materials, 2 (1975), 262.

13) Y. Inokuti, Y. Shimizu and H. Shimanaka: U.S. Patent, No. 4280856, (1981).

14) Y. Shimizu, Y. Obata, T. Imanaka and Y. Iida: Japanese Patent, No. S58-13606, (1983).

15) Y. Inokuti, Y. Shimizu and H. Shimanaka: Bull. Japan Inst. Metals, 18 (1979), 632.

16) Y. Iwasaki, Y. Inokuti, H. Shimanaka and K. Fujimoto: Bull. Japan Inst. Metals, 18 (1979), 632.

17) G. Maeda, Y. Inokuti and Y. Ito: J. Japan Inst. Metals, 50 (1986), 869.

18) Y. Inokuti, C. Maeda and Y. Ito: J. Japan Inst. Metals, 50 (1986), 874.

19) O. Furukimi, A. Yamamoto, M. Imanaka and U. Nishiike: The 86th Conf. of the Instrumentation Comm., the Joint Research Society, ISIJ, Rep. No. 86-6-4, (Mar. 1984).

20) T. Taoka, E. Furubayashi and S. Takeuchi: Tetsu-toHagané, 54 (1968), 22.

21) K. Matsumura and Y. Kamata: Tetsu-to-Hagané, 58 (1972), 80. 